\title{
REFLEXIONES EN TORNO A LA DIDÁCTICA: IMPLICACIONES EN LA FORMACIÓN DOCENTE
}

\author{
Sandra Bejarano Gutiérrez' \\ María de los Ángeles Castro Villalobos ${ }^{2}$ \\ Ana María Hemández Segura ${ }^{3}$ \\ Ana Herrera Castro ${ }^{4}$ \\ Gabriela Oviedo Madrigal ${ }^{5}$ \\ Susana Ruiz Guevara ${ }^{6}$ \\ Rosario Vargas León ${ }^{7}$
}

El presenta artículo es producto de la lectura y análisis en torno a la conceptualización de la didáctica y sus implicaciones en la formación docente. Esboza antecedentes de la didáctica y planeamientos actuales sobre la temática. Además, incluye algunos presupuestos didácticos necesarios para considerar en la formación docente.
This paper derives from reading about and analyzing how didactics is conceptualized, as well as the its implications in teacher training. It presents background information for the area of didactics and current issues on the problems of the area. In addition, we include some didactic assumptions that are necessary to take into account in teacher training.

1 Licenciada en Educación Preescolar.

2 Licenciada en Filología.

3 Licenciada en Educación Preescolar.

4 Licenciada en Educación Especial.

5 Licenciada en Educación con Concentración en I y II Ciclos y Máster en Psicopedagogía.

6 Licenciada en Educación Preescolar y Máster en Administración Educativa.

7 Bachiller en Artes Plásticas y licenciada en Administración Educativa.

Todas las autoras son académicas de la División de Educación Básica del CIDE. 


\section{Antecedentes que enmarcan la reflexión}

Las diversas conceptualizaciones de la didáctica no han escapado a la historia, la evolución de sus significados a través del tiempo lo evidencia. Comenius (1994) en su Didéctica Magna imprime la primera huella en el siglo $\mathrm{XV}$, y quinientos años después, todavía se continúa en la búsqueda de una mayor compresión del concepto. A esa búsqueda nos aventuramos.

La clasificación que presentan Sáenz, Blázquez, Femández, López, Lorenzo y Molina (1997), identifica tres grandes corrientes en la historia de la didáctica: la clásica, la tecnología y la antididáctica. La primera de ellas -la didáctica clásicase divide en dos momentos básicos: el primero, el de la didáctica tradicional con su representante Comenius; se caracteriza por dar énfasis a los contenidos como el elemento primordial de la enseñanza, bajo el protagonismo del docente.

El segundo momento de la didáctica clásica se da a principios del siglo $X X$, con el surgimiento de la Escuela Nueva que contrasta con la didáctica tradicional, por el énfasis que ofrece, ya no es el contenido el centro de la enseñanza, sino que el docente se convierte en el protagonista de los procesos de enseñanza y aprendizaje.

El debate continúa y a mediados del siglo XX el auge de la tecnología y sus avances se refleja en una didáctica tecnológica, en la búsqueda de una propuesta que responda a los cambios generados por las exigencias sociales y culturales de la época. Esta segunda corriente contempla como elementos básicos: la programación, ejecución y evaluación, cuyo fin es operacionalizar los objetivos para racionalizar recursos, tiempo y esfuerzo con miras a optimizar los procesos de enseñanza y aprendizaje. (Sáenz et al; 1997).

Ambas corrierites conviven por décadas, sin embargo, en los últimos tres decenios -fines del siglo XX-el marco de análisis de la didáctica ha venido transformándose: de circunscribirse a la didáctica clásica, pasa a teorizar sobre la enseñanza, en los 80 , cayendo en un reduccionismo marcado por la racionalidad técnica; hasta llegar a la década de los 90, en donde algunos estudios e investigaciones ofrecen nuevos aires que fortalecen el desarrollo teórico de la didáctica. Esto da como resultado el surgimiento de una tercera corriente denominada la antididáctica.

Con los aportes de Gramsci, Althouser y la Escuela de Frankfurt, (Ayuste, Flecha, López Palma, Lleras, 1998) se incorpora un elemento fundamental en el debate pedagógico: la toma de conciencia de la incidencia de los contextos sociohistóricos en las "prácticas de enseñanza".

De esta manera, el planeamiento que surge es el de la antididáctica,-tercera corriente en la clasificación de Sáenz-cuyo fundamento sociopolítico tiene como 
Aplicado a la realidad de las aulas, se requiere considerar que los sujetos que las conforman, están en constante cambio e influidos por sus contextos y por lo tanto, permanentemente resignificando sus acciones. De esta manera, una aula es un espacio dinámico donde no es posible aplicar "recetas" o "métodos" como si se tratara de un grupo homogéneo, estático, previsible. Es necesario -como actor docente del grupoabocarse a interpretar lo que cada persona del grupo quiere comunicar con sus acciones para realmente comprender lo que está sucediendo en ellas.

proposito la lucha por el cambio de la estructura de dominación, por medio de la accion, reflexión y concienciación. Así, la antididáctica asume una posición crítica social y of rece una gama de reflexiones sobre los problemas sociales actuales. En América Latina, Paulo Freire es uno de los principales exponentes de csta posición crítica, su discurso se organiza en torno a la reconstrucción social, visualizando la enseñanza como una actividad política. (Ayuste et al, 1998).

\section{Líneas de pensamiento contempo- ráneas y su relación con el concepto de didáctica en los últimos tiempos}

Las líneas de pensamiento que han marcado el rumbo del conocimiento en los últimos tiempos ofrecen un marco referencial y sobre todo, comprensivo alrededor de la conceptualización de la didáctica en nuestros días. De ahí la importancia de realizar un rápido recorrido por dichas líneas, para visualizar su aporte a la discusión y reflexión en torno a la didáctica y sus implicaciones en las prácticas educativas.

A principios del siglo $\mathrm{XX}$, a nivel mundial, se venía dando un cambio paradigmático alrededor del concepto de la ciencia. El año 1920 marca el comienzo con el Círculo de Viena, movimiento filos6fico con ciertas características particulares, entre ellas, concebir la construcción de ideas no como un acto individual, sino colectivo. Esto que parece fácil de decir, vendría a transformar el concepto de conocimiento, rcconociendo que en la producción de conocimiento, la interacción, el grupo y el dialogo juegan un papel fundamental. Las implicaciones que estos elementos tienen para la didáctica de nuestros tiempos son indiscutibles.

Uno de los representantes más críticos del Círculo de Viena fue Popper, (citado en Sarramona, 1986) personaje que revoluciona las creencias y concepciones de los científicos de la época, al plantear otro tipo de perspectiva para comprender y explicar la rcalidad. 
Su idea radica en visualizar a un sujeto de conocimiento -persona que intenta comprender y explicar la realidad- que además de la "objetividad" que pueda manifestar en sus indagaciones, en sus planeamientos, también puede subyacer la subjeti vidad, o sea, se concibe a un sujeto que capta y decide sobre los hechos, y que no solo los piensa racionalmente.

Con esta declaración, Popper le está dando un espaldarazo a la noción de ciencia, entendida como la construcción de una teoría sistemática de la naturaleza del hombre y la sociedad y pone sobre el tapete la significatividad que para cada sujeto tiene la realidad que pretende captar, comprender, transformar.

De esta manera, el conccpto de verdad absoluta divulgada durante la ciencia por años, queda en entredicho y rcscata una verdad relativa, cambiante, impredocible. marcada por la mirada del sujeto que la observa e interactúa en ella. La didáctica debe rescatar de esta línca de pensamiento la subjetividad y significatividad que ticne cada una de las acciones de los actores implicados en las prácticas educativas.

Una propuesta emergente que va a venir a influir en las concepciones y prácticas, la propone Kuhn (1987) en el marco del pensamiento histórico. Dicho movimiento concibe al sujeto de conocimiento desdc otra perspectiva, como un sujeto que ha ido construyendo a lo largo de la historia diversos modelos para interpretar la realidad; a estos modelos los ha denominado "paradigmas". Rescatamos de este movimiento la conccpción de un sujeto histórico en constante dílogo con el entomo y con necesidad de ir proponiendo nuevos marcos interpretativos, para comprender los cambios que se van sucediendo.

Otra línea de pensamiento que aporta a la comprensión de la realidad y de las construcciones teóricas, la constituye la escuela de Frankfurt, en la figura de Habermas (Ayuste et al, 1998), filósofo interesado en la autorreflexión de los agentes humanos, situacioncs, nociones. El aporte que ofrece para comprender la realidad es concebir la ciencia desde la perspectiva histórica hermenéutiça, es decir, de encontrar el sentido a los hechos, de interpretar lo que va sucediendo.

Ya no basta con explicar o comprender la realidad desde afuera, desde lo objeti vo, es necesario introducirse en la subjctividad, para encontrar el significado que para los sujctos tienen los hechos que succden. La implicación de esta línea de pensamiento en la didáctica es esencial, pucs considcra la subjetividad que tiene cada individuo.

Aplicado a la realidad de las aulas, se requiere considcrar que los sujetos que las conforman, están en constante cambio e influidos por sus contextos y por lo tanto, permanentemente resignificando sus acciones. De esta manera, una aula es un espacio dinámico donde no es posible aplicar "recetas" o "métodos" como si se tratara deun grupo homogéneo, estático, previsiblc. Es nccesario -como actor docente del grupo- abocarse a interpretar lo que cada persona del giupo quiere comunicar con sus acciones para realmente comprender lo que está sucediendo en ellas. 
En síntesis, consideramos que los aportes que han proporcionado estas líneas de pensamiento -Popper, Kuhm, Habermas- relativizan el concepto de ciencia, al dejar de considerarla como una verdad total, absoluta, a cambio de una verdad parcial, temporal, en constante transiormación. Además se rescata de sus propuestas, la noción de colectividad y subjetividad en la construcción de conocimientos, de la significatividad e interpretación de las acciones, del contexto y de las situaciones. De esta manera, la opción que se tome frente a cualquier concepto ya no puede ser la misma que antes. La conceptualización de la didáctica comienza a resignificarse.

\section{Concepto de didáctica}

La complejidad del marco teórico y referencial que acompaña a la didáctica en estos tiempos, no provicne únicamente, de la confluencia de las líneas de pensamiento antes mencionadas, ni de la diversidad de puntos de vista, sino también, de su objeto y trascendencia.

Es así como, de la interpretación que le demos a la didáctica dependerá nuestras prácticas educativas, de ahí lo crucial de encontrar claridad conceptual, claridad que debe pasar por una revisión de nuestro concepto de mundo, de la propia vida, del conocimiento, de los valores, de cómo se aprende, de cómo se organiza la sociedad.

Todo lo anterior hace complejo el marco conceptual de referencia, porque en la práctica de la enseñanza tiene lugar el cruce de todos esos supuestos, que son los que en definitiva fundamentan el propio objeto de estudio de la didáctica; mediatizada, además, por diversos enfoques filosóficos (acerca del hombre y su vida), enfoques epistemológicos (acerca de la naturaleza del conocimiento), enfoques psicológicos (acerca de cómo aprende el ser humano) y enfoques sociológicos y culturales (acerca del tipo de sociedad que se pretende).

Es por ello, que la didáctica en este nuevo milenio debe partir de una visión multidimensional de la práctica de la enseñanza educativa. De este modo, las diversas concepciones sobre la didáctica pueden contribuir a identificar elementos que posibilitan clarificar nuestra propia interpretación.

Para Alvarez (1997) didáctica:

(...)es la ciencia que estudia el objeto del proceso educativo: enseñanza-aprendizaje y posee las características de un sistema teórico: conceptos, leyes y una estructura particular de sus componentes que determinan una lógica interna, en la cual intervienen condiciones sociales, si bien externas al objeto mismo. (p.15). 
Para Díaz Barriga (s.f.) la didáctica "es una disciplina muy peculiar que históricamente se estructura para atender los problemas de la enseñanza en el aula". (p.4).

Para Bolaños y Zúñiga (1998) la didáctica se ha venido comprendiendo de diversas maneras, para dichas autoras, la didáctica, en su concepción revitalizada se asume como la disciplina teórico-práctica que orienta la labor de enseñanza del docente en relación con el aprendizaje del educando, posee un campo semántico propioy posibilita una reflexión dentro de parámetros epistemológicos, científicos y de influencia social. (p.l).

Al confrontar las concepciones anteriores, con las que cada uno de nosotros puede tener sobre la didáctica, e intentar una concepción integradora, nos hallamos ante una disyuntiva para llevarla a cabo, pues en cada una de ellas subyace una concepción de mundo, de persona, de conocimientos, de intenciones; supuestos que configuran concepciones de didácticas diversas.

Al encontramos frente a una diversidad conceptual es importante como lo recomienda Díaz Barriga (s.f) tomar una "postura dialógica" frente a ellas.

De esta manera, más que un planteamiento conceptual de didáctica, la propuesta a la que llegamos es el de ofrecer una serie de aspectos orientadores hacia los que debería encaminarse la reflexión del concepto de didáctica en la formación docente, para que cada uno de los actores - estudiantes en procesos de formación y profesores- analice, cuestione, sistematice su propia práclica pedagógica para encontrar su postura didáctica.

Como una aproximación a los aspectos a los que es necesario prestarle atención, identificamos los siguientes: la didáctica es una disciplina teórico práctica que debe conducir a la reflexión contextualizada de los procesos interactivos de enseñanza y aprendizaje, tanto en el ámbito intrapersonal, como interpersonal, propiciando la sistematizacion de la práctica pedagógica, para su significación e interpretación, así como su mejoramiento y transfommacion.

\section{Implicaciones en la formación docente}

De la reflexión y análisis de lo expuesto anteriormente, planteamos las siguientes implicaciones didácticas en la formación docente.

1. Relación teórico práctica: con respecto a la relación teoria y práctica existe una gran discusión, la confrontación de ambas y su casi irreconciliable vínculo ha dado al concepto de "praxis" la posibilidad de unificar estos dos aspectos.

En la fundamentación de los planes de estudios de la División de Educación Básica (2000), el concepto de praxis es entendido como "un proceso de acción y 
de reflexión, en diálogo permanente con la realidad y con los fundamentos que permiten su lectura y desarrollo" ( $p .24)$. Decir que la didáctica es una disciplina de la praxis educativa es considerar que el vínculo entre teoría y práctica se produce en la interacción entre el contexto y los participantes del proceso educativo, mediatizado por un constructo teórico que genera reflexión, análisis y reelaboración de la experiencia misma.

2. Didáctica reflexiva: el proceso reflexivo fundamental, tanto en la formación docente como en su ejercicio profesional implica un "volver hacia atrás"; en el sentido de revisar y repensar lo que hemos hecho en nuestras prácticas de enseñanza, apoyados en marcos teóricos que nos ayuden a encontrar el sentido de dichas prácticas y comprender la realidad.

La caracterización de un docente reflexivo que propone Schön (citado en Contreras 1999, p.77) es aquel que actúa sobre situaciones que son inciertas, inestables, singulares y en las que hay conflictos de valor. La distinción que hace entre "conocimiento en la acción" y "reflexión en la acción" es interesante de rescatar para tomar conciencia de nuestra práctica educativa.

"Nomalmente nuestra práctica cotidiana está asentada en un conocimiento tácito, implícito, sobre el que no ejercemos un control específico. Hay una serie de acciones que las realizamos espontáneamente sin pararnos a pensar en ellas antes de hacerlas. Son comprensiones de las cosas o competencias que hemos interiorizado de tal forma que nos sería difícil descubrir el conocimiento que implícitamente revelan estas acciones. Muchas veces ni siquiera somos conscientes de haberlas aprendido, simplemente nos descubrimos haciéndolas". (p.77). A esto Schön le llama "conocimiento en la acción", (Schön citado por Contreras, 1999).

Por otro lado, también es normal que en ocasiones ante algo inusual, nos distanciamos de la situación habitual y se piense sobre lo que se está haciendo; Schön le llama: "reflexión en la acción". (Schön citado por Contreras, 1999).

En estas situaciones, "conocimiento en la acción" y "reflexión en la acción" no se es dependiente de teorías o técnicas específicas, sino que entra en escena una relación dialógica entre el docente y el entomo que le posibilita una nueva manera de plantear el problema y decidir qué es lo que debe hacer.

Cualquiera que sea el camino "conocimiento en la acción" o "reflexión en la acción", el proceso de reflexión es lo que nos permite tomar conciencia de nuestra práctica pedagógica y de darle significación.

3. Didáctica contextualizada: consideramos que es fundamental para la compresion del hecho educativo estar consciente de todas las instancias que entran en juego: comunidad, cultura, familia, docentes, estudiantes, lo que en el plan de estudios se señala como diálogo permanente con la realidad.

Cuando Alvarez (1997, p.22) expresa que "la didáctica es una disciplina, teórica, histórica y política" alude a una concepción que se nutre de lo dinámico 
y cambiante de la sociedad, asignándole a la didáctica un carácter social, es decir, contextualizada. De esta manera, la singularidad de las situaciones educativas es una de las ideas que enfatiza Stenhouse (citado por Contreras, 1999, p. 84): "cada clase, cada alumno y cada alumna, cada situación de enseñanza refleja características únicas y singulares". Las acciones de enseñanza y aprendizaje poseen su significatividad propia, no se puede saber lo que es, o será una situación de enseñanza hasta que no se lleve a cabo; de ahí que Stenhouse enfatice que lo importante es atender las circunstancias que cada caso presenta y no pretender la uniformidad de los procesos educativos de los niños y las niñas.

4. Didáctica participativa: el proceso de enseñanza y aprendizaje es un proceso social y como tal interactivo, que debe propiciar la reflexión y el análisis entre los actores sociales.

5. Didáctica comunicativa: la transmisión de experiencias, conocimientos y actitudes en un grupo lleva a la identificación de éste. La comunicación de significados con intencionalidad conlleva a apropiarse de un discurso, de unas ideas, de una posición. Es importante por ello que las prácticas de enseñanza sean objeto de discusión y reflexion entre los futuros docentes. Un docente que reflexiona su práctica, la analiza, puede estar en mayor capacidad de mejorar sus cualidades pedagogicas, de renovar e innovar, de tomar decisiones, de proponer, de ser un docentc que transforme y mejore su quehacer pedagogico.

En conclusión, consideramos que la reflexión sobre la didáctica debe ser tarea de todo docente, que lo conduzca a la búsqueda de puntos de encuentro o desencuentros tebricos, a descubrir la diversidad de significados en términos similares, a dejar de creer en la verdad científica, a traer a discusión la complejidad de los contextos implícitos en el acto didáctico, a confrontar posturas tebricas divergentes.

Todo ello significa una nueva manera de abordar los procesos de enseñanza y aprendizaje en la formación docente, que más que llegar a obtener un producto final, como podría ser ei construir un concepto de didáctica, se vivan procesos didácticos en la búsqueda inalcanzable del concepto de didáctica.

\section{Referencias}

Álvarez, R. (1997). Hacia un curriculo integral y contextualizado. Honduras: Editorial Universitaria.

Ayuste, A., Flecha, R., L6pez Palma, F., Lleras, J. (1998). Planteamientos de la pedagogía critica. Comunicar y transformar: Barcelona: Editorial Grao. 
Bolaños, R. y Zúñiga, I. (1998). Maestría en Formacion docente con mención en didáctica. Propuesia Universidad Nacional. Centro de Investigación y Docencia en Educación. División de Educación Básica. Documento impreso.

Comenius, J.A. (1994). Didáctica Magna. México: Editorial Porrúa.

Contreras, D. (1999). La autonomía del profesorado: Madjid Morata.

Díaz Barriga, A. (1997). Didacrica y curriculum. Editorial Piado, Ibérica.

Díaz Barriga, A. (s.f.) La investigación en el campo de la didactica: modelos historicos. Documento en línea.

División de Educación Básica. (2000). Plan de estudios en Pedagogía con énfasis en l y ll ciclos de la Educacion General Basica. Heredia. Universidad Nacional. Centro de Investigación y Docencia en Educación (CIDE).

Elliot, L. (1996). El cambio educativo desde la investigacion accion. Madrid: Editorial Morata.

Freire, P. (1997). Pedugogia de la auıonomía. México D.F.: Siglo XXI Editores.

Kuhn, T.S. (1987). La estructura de las revoluciones cientificas. México D.F.: Fondo de Cultura Económica.

Martín Molero, F. (1999). La didactica ante el tercer milenio. Madrid: Editorial Síntesis.

Sáenz, O., Blázquez, F., Fernández, P., López, M., Lorenzo, M. y Molina, S. (1997). Didactica general. Madrid: Amaya.

Sarramona, J. y Salomó, M. (1985) "La pedagogía hoy" En: ¿Qué es la Pedagogia? Una respuesia acıul. Barcelona: CEAC. 